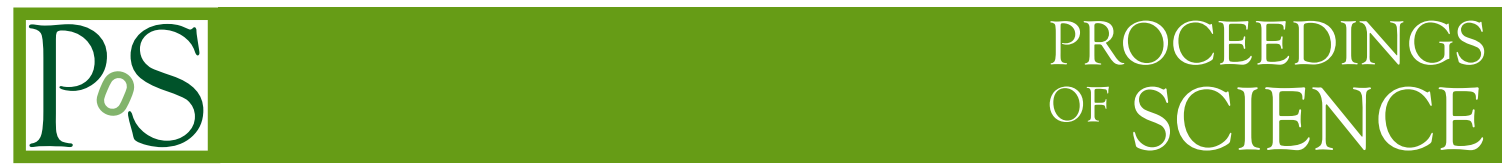

\title{
Welcome Remarks
}

\section{Joachim Trümper}

Max-Planck Institute for extraterrestrial Physics

E-mail: jtrumper@mpe.mpg.de

In this opening remark, I shortly sketch the history of Gamma-ray astronomy and Gamma-ray Burst physics at the Max-Planck Institute for extraterrestrial Physics.

Gamma-Ray Bursts 2012 Conference - GRB2012,

May 07-11, 2012

Munich, Germany 
It is a pleasure for me to welcome you on behalf of our institute, the Max-Planck Institute for extraterrestrial Physics (MPE), and its High-Energy Astrophysics group. We are very happy that the Fermi/Swift Gamma-Ray Burst (GRB) conference came to Munich this year. It is very nice to see here many old friends, and even many more new faces. Well, I have only a few minutes time and I thought it would be most appropriate to sketch very briefly the history of Gamma-ray astronomy and GRB physics at MPE.

The MPE was founded in 1963 and started with ionospheric and magnetospheric research under Reimar Lüst and Gerhard Haerendel. Three years later, Klaus Pinkau joined MPE and imported the gamma-ray activities with balloon experiments. Our first successful gamma-ray satellite project was COS-B, and after that Klaus pushed the MPE involvement in CGRO before he left MPE in 1980. Volker Schönfelder became his successor as the head of the group. He was PI of COMPTEL, while Gottfried Kanbach and Hans Mayer-Hasselwander were involved in EGRET. The nineties with CGRO and our ROSAT up in orbit were busy times for the High Energy Astrophysics at MPE. But the next decade after the millennium was not worse, since the Gamma-ray group participated in INTEGRAL and got involved in Fermi with the Gamma-ray Burst Monitor (GBM) detectors.

Turning now to Gamma-ray bursts, it is great that two of the early pioneers are here with us - Tom Cline, who was involved in the discoveries with the Vela satellites and Rafail Aptekar from the Mazets group who followed up with Kosmos 461. At MPE the first gamma-ray burst was observed with the TD1 satellite. Although the operation of the spark chamber was severely affected by locally produced gamma-rays, the anticoincidence detector of experiment S133 picked up a strong burst on May 14, 1972 which was reported by Wolfgang Voges and Klaus Pinkau at the Conference on Transient Cosmic Gamma and X-Ray Sources at Los Alamos in 1973. Stimulated by the Vela results which were published in 1973, Michael Sommer from MPE started a balloon experiment together with Dietrich Müller from Chicago. In August 1976, they had a spectacular transatlantic balloon flight from Sicily to Massachusetts, with a duration of 107 hours. The payload was recovered but burned out four days later on a transport vehicle. From the ashes they were able to recover the film. After developing it, they found several solar bursts and one cosmic burst which gave rise to an ApJ Letters paper. However, this paper could not make a big splash anymore, since at that time gamma-ray burst data were already coming from interplanetary spacecrafts like IMP 6 and 7 and Helios-B, which was built here in Munich by Messerschmitt-Bölkow-Blohm (MBB). Last night, Tom Cline told me about his early visits to Munich for project meetings at MBB.

During those years, we had two visitors at MPE who later made great careers in the field. One was Neil Gehrels who in 1974 worked for half a year in Dieter Hovestadt's group, and the other was a young Greek student by the name of Chryssa Kouveliotou who came to us in 1978, did her $\mathrm{PhD}$ work on solar gamma-ray bursts at MPE, and got her degree from the Technical University Munich in 1981.

In October 1989, I had the chance to visit the GDR academy institute for Cosmic Research in East Berlin. It was two weeks before the fall of the Berlin wall, which had made scientific contacts between East and West German scientists practically impossible for almost thirty years. In that institute I met Jochen Greiner and was impressed by his systematic work on defects of photographic plates which had led others to wrong claims of optical identifications of GRBs. Some time later, we invited him to join MPE, where he later became the leader of the gamma-ray group after Volker 
Schönfelder's retirement. One of his major contributions is the very successful GROND instrument - and of course his organization of this meeting.

Support for gamma-ray burst astronomy came also from the X-ray group of the MPE. I knew Livio Scarsi since my cosmic-ray times in the 1960's. In the 1980's we got involved in the ItalianDutch SAX satellite by supporting the X-Ray mirror development and telescope calibration with tests in our PANTER test facility. Starting in 1997, the epoch-making BeppoSAX observations discovered the X-ray afterglows of gamma-ray bursts, which in turn led to the first optical identification by Jan van Paradijs who was a good friend of mine. ROSAT ToO observations organized by Jochen Greiner improved the BeppoSAX positions for a number of GRB afterglows down to 10 arcsec, paving the way for the recognition of optically dark bursts.

It is perhaps less well known that our X-ray group has also contributed to Swift. Its X-ray telescope was originally developed jointly by the Leicester group and us for the first Russian SpektrumRöntgen-Gamma project which collapsed due to the turbulent political changes in Russia in the nineties. Bernd Aschenbach from our group did the optical design of the JET-X telescope, and Heinrich Bräuninger did the X-ray mirror testing in our test facility. It was a great idea to use this telescope for Swift, which returned to MPE in September 2002 for final calibrations.

Last but not least - our institute got also involved in Fermi. It began with a phone call from Jerry Fishman to Volker Schönfelder, asking him whether MPE would be interested in contributing the detectors for the burst monitor. Volker agreed, after we had checked the feasibility in the institute, leading to a very fruitful collaboration, which from our side was led by Giselher Lichti and Andreas von Kienlin. I'm looking forward to hearing about the scientific highlights of the GBM later this morning.

Let me close by saying, that we at MPE are very happy to see Munich being the Center of the GRB world for a few days. I wish all of us an interesting meeting and our visitors an enjoyable time in Munich.

Thank you very much! 